\title{
El impacto económico en la industria maquiladora y en la región fronteriza del norte de México debido al alza de $100 \%$ del salario mínimo
}

\section{The Economic Impact on the In-Bond Industry and on the Northern Border Region of Mexico due to the $100 \%$ Increase of Minimum Wage}

\author{
Noé Arón Fuentes* (D) https://orcid.org/0000-0002-9254-8107 \\ Alejandro Brugués Rodríguez ** (D) https://orcid.org/0000-0001-5964-7974 \\ Gabriel González König ${ }^{\star \star *}$ (iD https://orcid.org/0000-0003-4897-9687 \\ Jorge Carrillo Viveros ${ }^{* \star * \star}$ (D) https://orcid.org/0000-0002-2008-8818
}

\begin{abstract}
Resumen
El objetivo del estudio es medir el efecto en la economía de la región fronteriza del incremento del salario mínimo a partir de la declaración de la zona libre de la frontera a principios de 2019. La medición se basa en un enfoque de precios del modelo de insumo-producto siguiendo la propuesta de Park (2006) y en una estimación propia de la matriz de insumo-producto para la región. La evaluación consideró el alza de 100\% del salario mínimo que favoreció alrededor de 63I mil de trabajadores - I46 mil de la industria maquiladora y 485 mil en el resto de los sectores - . Los resultados del modelo muestran un incremento de $2.9 \%$ sobre la inflación, y asociado a ello se espera un ajuste a la baja de la producción de I\%, del personal ocupado de $1.7 \%$ y del valor agregado de I.I\%. En particular, la industria maquiladora es menos sensible a estas variables clave, ya que el salario medio pagado por ellas era más cercano al salario mínimo establecido. Adicionalmente, hay un impacto positivo en el ingreso de los trabajadores de salario mínimo en la industria maquiladora, pues ahora ganan $6.8 \%$ más, y del resto de los sectores, ya que ahora ganan $9.7 \%$ más. Si se toma en cuenta lo anterior, se puede establecer que la política laboral regional es una medida de bajos efectos inflacionarios y de contracción, aunque ligera, en la actividad económica, pero que mejora el poder adquisitivo de los que menos ganan.

Palabras clave: salario mínimo; industria maquiladora; insumo-producto.
\end{abstract}

Cómo citar: Fuentes, N.A., Brugués Rodríguez, A., González König, G., y Carrillo Viveros, J. (2020). El impacto económico en la industria maquiladora y en la región fronteriza del norte de México debido al alza de $100 \%$ del salario mínimo. región y sociedad, 32 , el 230. doi: $10.22198 /$ rys2020/32/1230

* Autor para correspondencia. El Colegio de la Frontera Norte, Departamento de Estudios Económicos. Carretera Escénica Tijuana-Ensenada Km. 18.5 San Antonio del Mar, C. P. 22560, Tijuana, Baja California, México. Correo electrónico: afuentes@colef.mx

** El Colegio de la Frontera Norte, Departamento de Estudios Económicos. Avenida Insurgentes Núm. 3708, Colonia Los Nogales, C.P. 32350, Ciudad Juárez, Chihuahua, México. Correo electrónico: abruguez@colef.mx

${ }^{* * *}$ Consultor independiente. C. del Mar Núm. 221, La Perla Residencial, Playas de Tijuana, C. P. 22505, Tijuana, Baja California, México. Correo electrónico: ggkonig@gmail.com

**** El Colegio de la Frontera Norte, Departamento de Estudios Sociales. Carretera Escénica Tijuana-Ensenada Km. 18.5, San Antonio del Mar, C. P. 22560, Tijuana, Baja California, México. Correo electrónico: carrillo@colef.mx

Recibido: 26 de junio de 2019

Aceptado: 5 de diciembre de 2019

Liberado: 23 de enero de 2020

\begin{abstract}
An the bord tion for the region. For the evaluation it was considthe $100 \%$ minimum wage increase, which fafre in-bond industry and 485 thousand in the the model sonomic sectors. The results coming from employed personnel; and I.I\% in the added value, all ated to that increase. In-bond industry, particularaverage salary paid in that industry is nearer to esminimum wage. In addition to that, there is a positive impact in the income of those in-bond industry workers earning minimum wage because now sectors receive $9.7 \%$ more money. Considering the 作 activity, but it enhances purchasing power of those who earn less.
\end{abstract}

Keywords: minimum wage; in-bond industry; input-output. 


\section{Introducción}

En diciembre de 2018 el presidente Andrés Manuel López Obrador publicó la Ley de Ingresos de la Federación (LIF) para el ejercicio fiscal de 2019. En particular, el paquete económico incluyó un conjunto de políticas fiscales y laborales focalizadas en los 38 municipios contiguos a la línea fronteriza entre México y Estados Unidos, y en los cinco del estado de Baja California - los 43 municipios denominados aquí región fronteriza norte (RFN) (LIF, Diario Oficial de la Federación, 28 de diciembre de 2018).

El paquete económico para la RFN considera la reducción de la tasa del impuesto al valor agregado (IVA) de $16 \%$ a $8 \%$ (rebaja de $50 \%$ para los intermediarios de la cadena productiva de la región); la reducción de la tasa del impuesto sobre la renta (ISR) de 30\% a 20\% (a los contribuyentes de la región que sean personas físicas o morales); y el aumento del salario mínimo fronterizo (SMF) de 88.36 a 176.72 pesos diarios (incremento de 100\%). Además, se proponen ajustes a los precios de los combustibles para equipararlos con los de la zona sur de Estados Unidos; y, también, se sugiere atender las carencias en los servicios urbanos básicos, el equipamiento de infraestructura y los espacios públicos.

En el paquete económico se juzga que el trato diferencial para la RFN tiene como fin incrementar su competitividad productiva e industrial, atraer inversión para generar más empleos, fomentar el consumo de sus habitantes, aumentar el comercio nacional y contener los flujos migratorios. Hay que destacar que la RFN tiene mayor apertura económica que el resto del país, también posee acceso diferenciado a los mercados internacionales de bienes y trabajo, que su grado de tercerización es alto (principalmente en turismo y comercio), y además desarrolla cultura, patrones de migración y procesos sociales propios. Cuenta también con relevantes sectores de actividad, como la industria manufacturera, maquiladora y de servicios de exportación (IMMEX). ${ }^{1}$ En pocas palabras, en el paquete económico se asume que la estructura económica industrial interna y la ubicación geográfica de la RFN constituyen una oportunidad para alcanzar los fines nacionales antes mencionados.

En 2019 el incremento de 100\% del SMF fue una fuente de conflicto laboral en la IMMEX. En particular, los trabajadores de Matamoros pararon labores y con el apoyo sindical comenzaron una huelga el 25 de enero. Su principal demanda era el incremento salarial -al menos de $20 \%$ en la industria en general- en correspondencia con el aumento del SMF y el pago de un bono anual de 32251 pesos, equivalente a un año de la diferencia del incremento del SMF. La movilización de los trabajadores tuvo su origen en el hecho de que el contrato colectivo del sindicato del sector de la IMMEX estipula que los incrementos salariales se realizarán conforme al aumento del salario mínimo. Debido a lo anterior, se generó la preocupación por el efecto del alza del nivel del SMF y por el efecto dominó en el resto de la distribución salarial formal fronteriza. Además, existe un interés manifiesto por analizar el impacto económico del alza en el nivel del

El Programa IMMEX autoriza a las empresas a elaborar, trasformar o reparar bienes importados temporalmente con el propósito de realizar una exportación posterior y/o venta nacional (Secretaría de Economía, 2011). 
SMF sobre variables clave, tanto en la IMMEX como en la RFN, debido al posible aumento de la inflación regional (Carrillo, Fuentes, Brugués y González-König, 2019).

Por esa razón aparece el interés en evaluar cómo se trasmite a cambios en precios regionales no sólo directamente el alza del SMF, sino también indirectamente el efecto dominó que se produce sobre el resto de la distribución salarial fronteriza. Además, importa el examen del impacto económico a corto plazo en la producción bruta, en personal ocupado y en valor agregado para la IMMEX y la RFN. Para ello, empleamos, en términos metodológicos, el enfoque de precios en el modelo insumo-producto.

El modelo de insumo-producto contiene un sistema de precios relativos implícitos o precios sombra. Se trata de aquellos que no corresponden a los del mercado. Los precios proporcionan una medida de consumo total de los insumos primarios. El supuesto de una economía competitiva, que elimina las utilidades en todos los sectores, implica que en ningún caso el precio de un bien o servicio puede ser mayor que el costo de producción. Además, que el principio de la eficiencia económica sugiere que tampoco el costo puede superar el precio, puesto que, en el caso contrario, el bien no se produciría. De este modo, el modelo registra los cambios de la relación de precios implícitos ante modificaciones en los impuestos, los salarios y el tipo de cambio (Cárdenas, 2006; Souto, 2002).

Los resultados basados en el modelo intersectorial de precios indican que el alza del SMF repercute en $2.9 \%$ sobre la inflación de la RFN -sin tomar en cuenta la reducción de los impuestos del IVA y del ISR-. En el caso del ingreso por trabajador, se tiene que con este aumento los empleados en la IMMEX de la RFN, en general, ganan $6.84 \%$ más que en el resto del país, mientras que los empleados que no pertenecen a la IMMEX en la RFN ganan un salario.

El texto se organiza en siete secciones aparte de la introducción. En la segunda sección se establece la iniciativa del alza al doble del SMF propuesta por el nuevo gobierno federal. En la tercera se revisa la literatura que trata el efecto del salario mínimo sobre la inflación, en la que se destaca el efecto dominó. En la cuarta se presenta el efecto de la trasferencia a precios e ingresos fronterizos derivado del aumento del SMF y su efecto sobre el resto de la distribución salarial. En la quinta se muestra el modelo insumo-producto de precios implícitos o sombra. En la sexta se esboza la elaboración de la tabla insumo-producto de la región fronteriza norte y de la del resto del país. En la séptima se muestran los resultados del impacto económico en la IMMEX y en la RFN debido a los cambios exógenos de los precios regionales. Al final, en la octava sección, se realizan conclusiones.

\section{Decreto del aumento en el salario mínimo fronterizo}

En diciembre de 2018, el presidente López Obrador anunció los criterios generales de política económica y el Proyecto de Presupuesto de Egresos de la Federación Ejercicio Fiscal 2019. De acuerdo con los criterios generales, en todos los 
municipios ubicados a lo largo de la línea internacional entre México y Estados Unidos y en los del estado de Baja California, se decretó un incremento del SMF de 88.36 a 176.72 pesos diarios (alza de $100 \%$ ).

En 2019 el decreto publicado del alza al doble del SMF generó fricciones laborales en la IMMEX localizadas en la región fronteriza norte. Específicamente, en la ciudad de Matamoros, Tamaulipas los empleados pararon labores y con el apoyo de su organismo sindical proclamaron una huelga hacia finales de enero. Su principal demanda era el incremento salarial -al menos de $20 \%$ en la industria en general- en correspondencia con el aumento del SMF y el pago de un bono anual de 32251 pesos, equivalente a un año de la diferencia del incremento del SMF. La demanda se basó en el contrato colectivo que estipula que en el sector industria los aumentos salariales están indizados al salario mínimo (Carrillo et al., 2019).

Ante ese aumento salarial, las empresas reaccionaron retirando bonos (de puntualidad y de productividad, vales de despensa y fondos de ahorro). Esta situación no fue aceptada por los trabajadores, quienes finalmente fueron representados por el sindicato en la negociación de los incrementos en salarios y prestaciones. A pesar de que este movimiento incluyó inicialmente a trabajadores de 45 maquiladoras y que luego se extendió a otras, hasta el mes de enero de 2019 las negociaciones sólo se han realizado empresa por empresa, tal como procede según la legislación. Ésta es una situación que pudo influir en que las demandas y los paros se extendieran hacia otros sectores, como los de tiendas departamentales, de autoservicio y a los trabajadores de recolección de basura de Matamoros.

Sin embargo, esta situación no se ha generalizado a lo largo de la RFN. La Asociación de Recursos Humanos de la Industria en Tijuana (ARHITAC) argumentó que en esa ciudad es difícil que haya una movilización similar a la de Matamoros, ya que, desde hace varios años, cerca de $70 \%$ de las empresas que tienen afiliadas paga un salario mayor (Martínez, 2019).

En tanto, la Asociación de la Industria Maquiladora y de Exportación (INDEX) de Tijuana indicó que ese aumento sólo tendría impacto en $20 \%$ de sus miembros, ya que el resto gana más de lo que el gobierno federal fijó como mínimo (Martínez, 2019).

En síntesis, existe una preocupación en cámaras y asociaciones empresariales regionales por el alza al doble del SMF debido a la posible existencia de impactos positivos en la inflación regional y de impactos negativos en la producción, la ocupación y el valor agregado, tanto en los sectores estratégicos (IMMEX) como en el resto de las actividades económicas de la RFN.

\section{Literatura sobre el efecto del salario mínimo sobre precios}

En la literatura internacional se reportan varios estudios sobre los efectos del salario mínimo realizados desde diferentes perspectivas. Al respecto, Gosling (1996); Johnson, (1983), y Lin y Yun (2016) han analizado los impactos sobre la distribución del ingreso; en Addison (1999) y Neumark (2002) se abordan los 
efectos sobre la pobreza; una reflexión general sobre los efectos se refiere en MaCurdy y O'Brien-Strain (2000); finalmente, Lemos (2004) aborda los efectos sobre los precios y expresa como ésta es una de las perspectivas menos estudiadas.

En la literatura mexicana podemos distinguir tres grupos de estudios centrados en el análisis del efecto del alza del salario mínimo sobre la inflación. El primer conjunto de estudios se basa en un ejercicio metodológico diseñado para analizar el traspaso de un cambio administrativo en el salario mínimo que afecta a un número limitado de trabajadores y que podría ocasionar efectos sobre los demás salarios, al que se denomina efecto dominó. El Banco de México (BANXICO, 2016), en su estudio titulado Salario mínimo e inflación, define ese efecto como "el traspaso que tienen los aumentos al salario mínimo sobre el resto de la distribución salarial, en especial sobre los ingresos de los trabajadores cuyo salario está por encima de dicho indicador."(p.11). El estudio señala que estimar ese efecto es relevante porque constituye uno de los principales canales de impacto que un incremento del salario mínimo puede tener tanto en la inflación como en el mercado laboral. Además, concluye que la presencia del efecto dominó constituye un riesgo importante para que incluso un incremento del salario mínimo de magnitud moderada tenga un efecto positivo sobre la inflación y negativo sobre la economía en general.

Hernández (2016), basado en dicho estudio, identifica el efecto del salario mínimo general (SMG) sobre el salario base de cotización (SBC) -denominado efecto dominó- y señala que el alza en el SMG aumenta el SBC de los trabajadores formales de la economía. También indica que este efecto varía a lo largo de la distribución salarial entre los trabajadores que reciben cuando menos una revisión salarial en el año; es decir, el SBC ocasiona un aumento en el corto plazo, tanto en el primer año como en el segundo. Y, por último, determina que el efecto disminuye poco a poco conforme el nivel salarial aumenta, pero se mantiene elevado para todos los grupos salariales considerados.

El segundo conjunto de estudios se centra en el efecto dominó analizando la dinámica entre el salario mínimo y las revisiones salariales. El estudio elaborado por el Gobierno de la Ciudad de México titulado Política de recuperación del salario mínimo en México y en el Distrito Federal; propuesta para un acuerdo nacional (2014), expone el efecto que tienen los cambios en el salario mínimo sobre las revisiones salariales reportadas. Moreno-Brid, Garry y Monroy-Gómez (2014) señalan que "la precondición jurídica para eliminar el efecto del salario mínimo [en la inflación] es la desindexación, es decir, la desvinculación del salario mínimo en tanto unidad de referencia de otros precios de trámites, multas, impuestos, prestaciones, etcétera" (p.13). Martínez (2019) establece que el salario mínimo en México es bajo. La causa es la visión monetaria que liga el salario mínimo con la inflación, lo cual motiva una política en la que el salario mínimo aumenta igual o menos que los precios en general. Concluye que no hay evidencia convincente de que tal causalidad exista. Además, Huesca y Llamas (2017), mediante una metodología no paramétrica, realizan simulaciones de incrementos salariales al doble y al triple en el país. Proponen que esa política laboral de mejora salarial en el país no sólo no sería inflacionaria, lo cual re- 
sultaría en una distribución más justa, sino que también reduciría la brecha de desigualdad salarial y del pago de renta de los perceptores.

El tercer conjunto de estudios está encabezado por la Organización Internacional del Trabajo (OIT), cuya investigación se titula El efecto del salario mínimo (efecto dominó/ efecto faro) en la distribución salarial de la economía mexicana (OIT, 2016). El estudio utiliza el episodio de homologación de las áreas geográficas de noviembre de 2012 para estimar, a partir de datos de la Encuesta Nacional de Ocupación y Empleo (ENOE), el efecto que un alza en el salario mínimo tiene sobre los salarios de los sectores formal e informal. A diferencia de los grupos de estudios anteriores, los resultados obtenidos en esta investigación no detectan la presencia del efecto dominó del salario mínimo sobre la distribución salarial en México.

En resumen, existen teorías y evidencias empíricas que muestran que bajo ciertas circunstancias los aumentos en el salario mínimo resultan y no resultan en aumentos de la inflación. Sin embargo, de acuerdo con los anteriores grupos de estudios, para que una política laboral de modificación del salario mínimo sea efectiva es necesario buscar modos de eliminar, minimizar o neutralizar el efecto dominó para evitar distorsiones sobre el mercado laboral. Consecuentemente, se podrá incrementar el nivel del salario mínimo en beneficio de los trabajadores sin ocasionar incrementos salariales generalizados desligados de las condiciones de la economía, de manera que no se incremente la inflación ni se afecte el funcionamiento del mercado laboral y la economía en general.

\section{Efecto trasferencia a precios fronterizos del aumento en el salario mínimo fronterizo}

El alza al doble del SMF favorece aproximadamente a 631 mil trabajadores -146 mil empleados en la IMMEX y 485 mil empleados del resto de los sectores económicos de la RFN- que perciben menos de dos salarios mínimos diarios.

Para calcular el efecto traspaso sobre precios regionales de esta política laboral, se utilizó un método que consiste en cuatro etapas (BANXICO, 2016). La primera etapa mide el efecto del SMF sobre los costos laborales. Para ello, se usaron los datos de la distribución de los trabajadores asegurados del Instituto Mexicano del Seguro Social (IMSS) y la masa salarial por rama de actividad. Los datos referidos se obtuvieron de la plataforma abierta disponible en la siguiente dirección: http://datos.imss.gob.mx/group/asegurados. La base de datos para los cálculos fue la correspondiente al mes de junio de 2018. La metodología para construir el incremento porcentual en la cotización del servicio de los trabajadores en el IMSS por rama de actividad consiste en: 1) partir del número de los asegurados totales en esa institución por rama de actividad, y dividir en los asegurados que perciben menos de dos salarios mínimos; 2) calcular la masa salarial promedio de la cotización del servicio del IMSS y la diferencia entre ésta y el diferencial para obtener dos salarios mínimos por día para los asegurados que perciben menos de dos salarios mínimos; y, 3) calcular el incremento 
del costo de cotización de los servicios del IMSS por sectores, en su mayoría de la IMMEX ,y para el resto de los sectores de la RFN en términos porcentuales, respectivamente.

En la figura 1 se advierte que los trabajadores de la IMMEX tienen de inicio mayores niveles salariales que el resto de los sectores económicos de la RFN. Por esta razón el alza de los costos laborales es de tan sólo 1.8\%, mientras que se produce un incremento en los costos laborales de $4.8 \%$ para el resto de los sectores económicos de la RFN.

Figura 1. Salario promedio diario de cotización, de noviembre de 2017 a octubre de 2018

- Cotización -IMMEX \$143.46 —Resto \$123.95 —SM Frontera Norte \$176.72

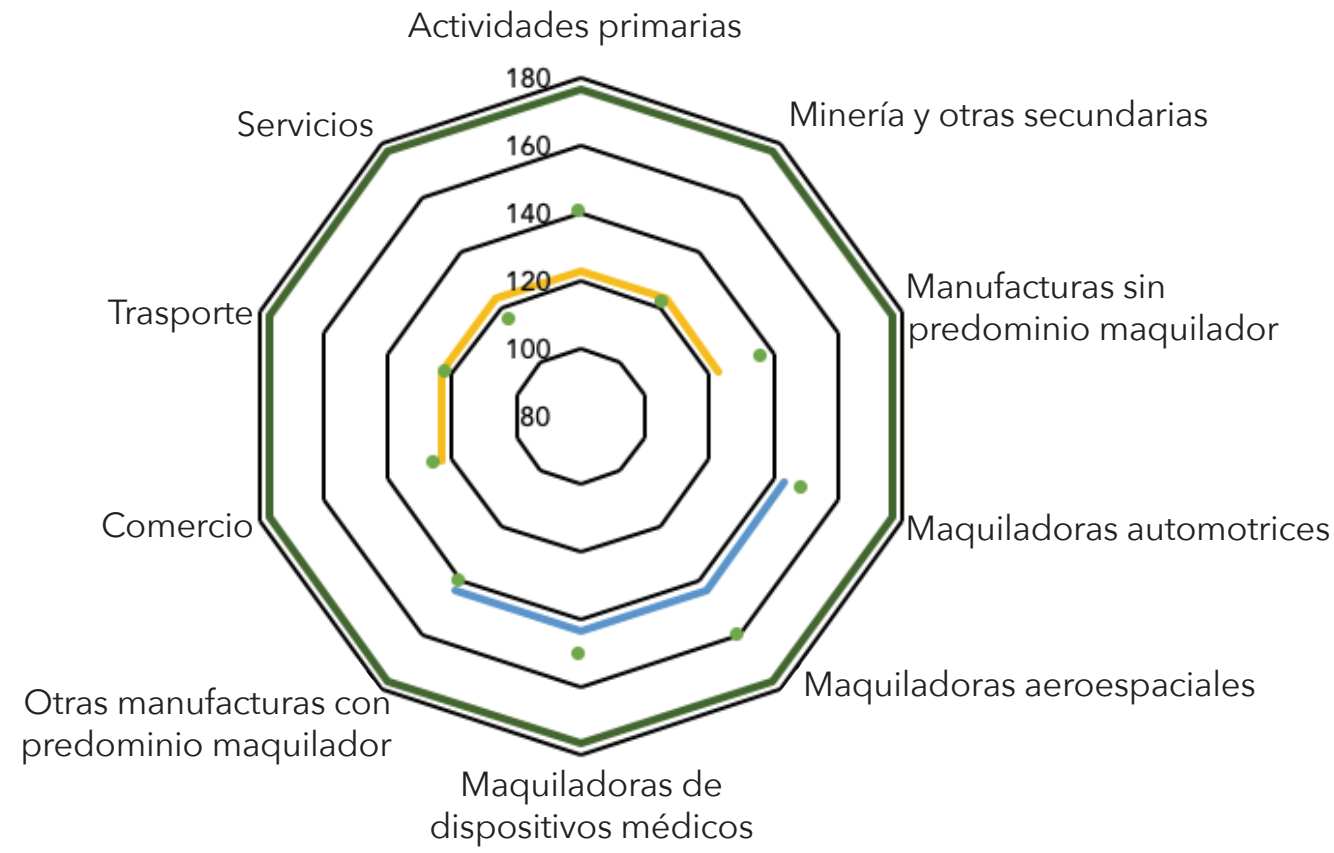

Fuente: elaboración propia con datos del Instituto Mexicano del Seguro Social, Datos abiertos, Conjunto de datos de asegurados, disponible en http://datos.imss.gob.mx/group/asegurados

La segunda etapa consiste en el traspaso que tiene el alza del SMF sobre el resto de la distribución salarial. Aquí debe notarse que puede existir un efecto dominó que implica un desplazamiento a la derecha de la distribución salarial, que podría inducir a las empresas regionales a alzar sus precios al consumidor ante un incremento generalizado en los costos laborales, a un aumento indirecto en los costos de bienes o servicios intermedios y a modificar otros márgenes, como podrían ser, por ejemplo, sus niveles de empleo formal. 
Para calcular el efecto dominó se usaron los datos del IMSS entre los meses de diciembre de 2018 y enero de 2019. En las figuras 2 y 3 se muestran, para IMMEX y el resto de los sectores de la RFN, las distribuciones de los asegurados del IMSS por niveles de SMF entre las dos fechas anteriores. En ellas se aprecia un desplazamiento de la distribución de enero de 2019 hacia la derecha, lo que implica un incremento en los niveles de ingresos de las personas que ganaban menos de dos salarios mínimos diarios. Este desplazamiento hacia mayores niveles de ingreso se combina con una distribución más compacta en términos de su amplitud, como se aprecia en las figuras de "cajas y bigotes" debajo de la gráfica de barras.

Figura 2. Industria maquiladora frontera norte.

Distribución de asegurados por rangos de salario mínimo

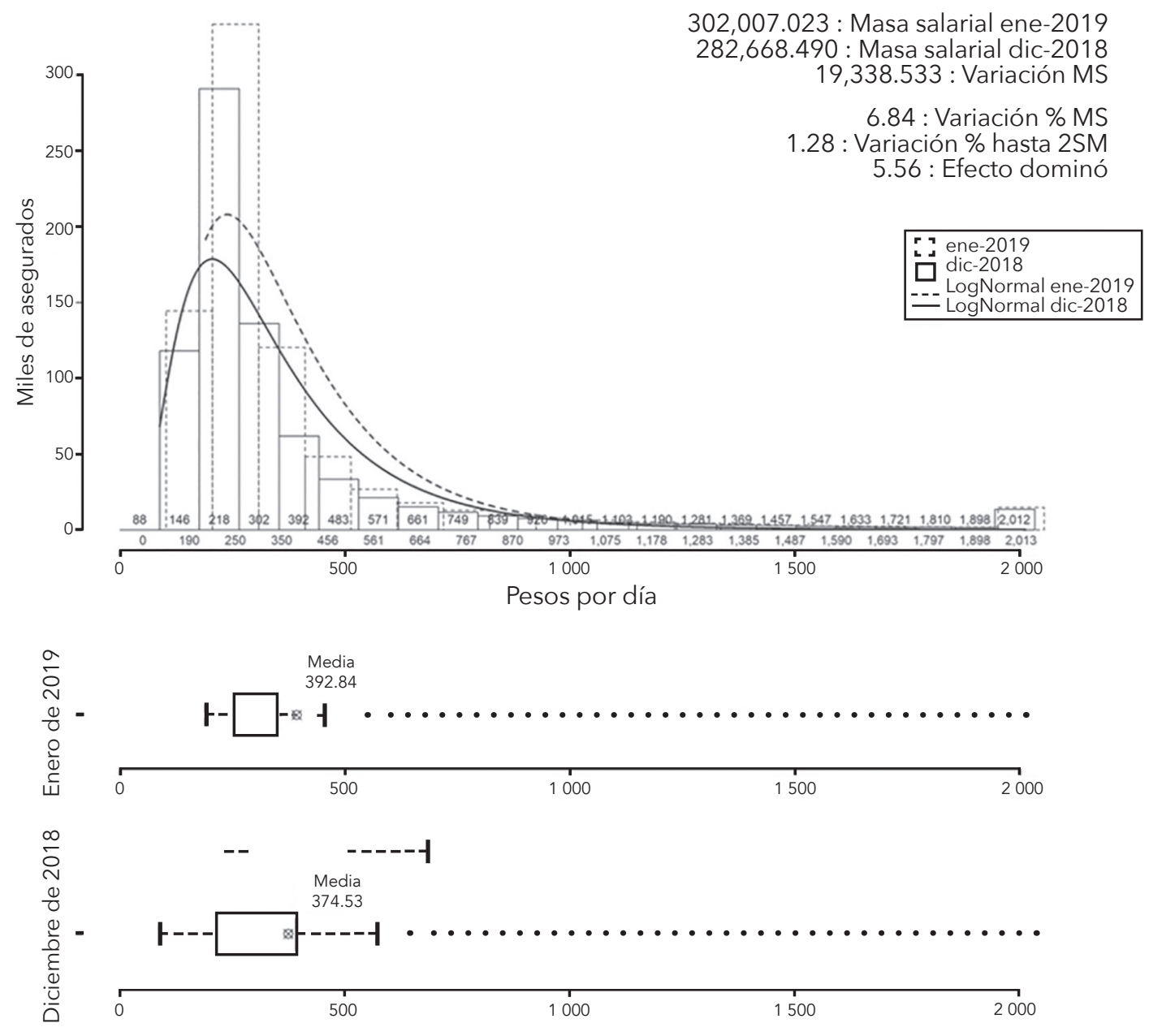

Fuente: elaboración propia con datos del Instituto Mexicano del Seguro Social, Datos abiertos, Conjunto de datos de asegurados, disponible en http://datos.imss.gob.mx/group/asegurados 
Como se muestra en la figura 2, al analizar el comportamiento para el caso de la IMMEX, en la que se benefician 146 mil trabajadores, se encontró que el efecto dominó es de 0.0684 (6.84\%); es decir, en promedio, un incremento del salario mínimo real de $1 \%$ ocasiona un aumento de 0.0684 en las revisiones salariales de los trabajadores del sector formal de la economía. Luego un aumento del SMF en $100 \%$ ocasionará un $6.84 \%$ de incremento en las revisiones salariales de la IMMEX. A este efecto se le conoce como el alza del salario base de cotización (SBC).

Figura 3. Frontera norte. Distribución de asegurados por rangos de salario mínimo

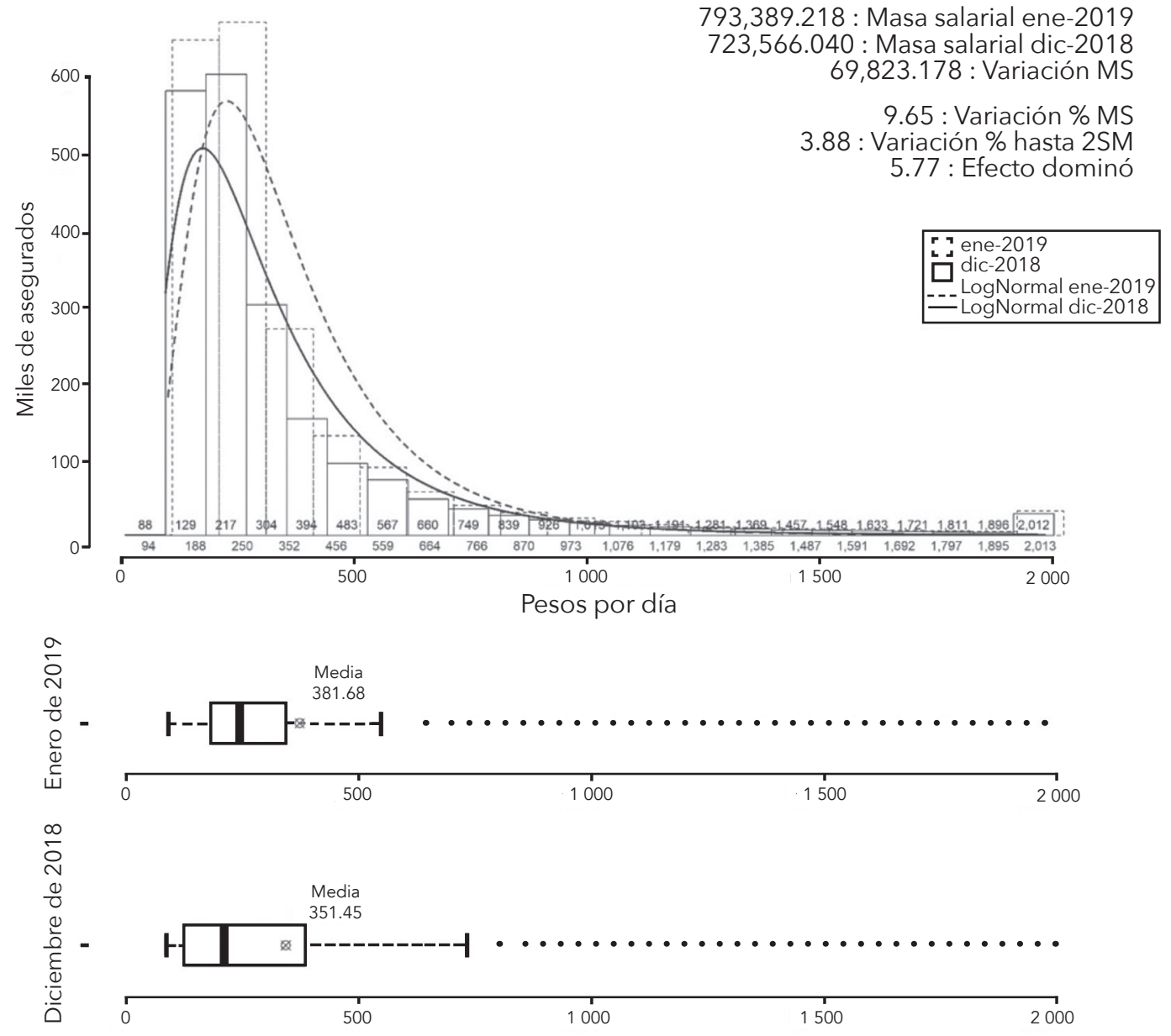

Fuente: elaboración propia con datos del Instituto Mexicano del Seguro Social, Datos abiertos, Conjunto de datos de asegurados, disponible en http://datos.imss.gob.mx/group/asegurados 
Como se aprecia en la figura 3, al analizar el comportamiento para el caso del resto de los sectores de la RFN, se tiene que se benefician 485 mil trabajadores y se encuentra que el efecto dominó es de 0.0965 , es decir, en promedio, un incremento del SMF de $100 \%$ ocasiona un $9.65 \%$ de incremento del SBC de la RFN.

En la tercera etapa se establece el grado y el tiempo de trasferencia completa a precios regionales del aumento en el SMF. Aquí se supone que, al igual que en el país (y de acuerdo con Banxico, 2016), un alza del SBC de 1\% produce un aumento de $0.18 \%$ en la inflación 12 meses después de que dicha alza ocurre. Finalmente, en la cuarta etapa se combinan los resultados anteriores para examinar el efecto de trasmisión completa que un incremento del SMF tiene sobre la inflación. Así, se infiere que una alza de $100 \%$ en el SMF repercute en $2.9 \%$ $\left(0.1634^{*} 0.18\right)$ sobre la inflación de la RFN 12 meses después del aumento al SMF, sin considerar otros efectos de retroalimentación, es decir, sin considerar los beneficios de la reducción en los impuestos del IVA e ISR en la RFN si y sólo si se mantienen constantes otras variables (tipo de cambio, deuda interna).

Sin embargo, en el caso del ingreso por trabajador, el alza del SMF tuvo efectos positivos y significativos. Debido al incremento en el SMF, los trabajadores de la IMMEX en general ganan ahora $6.84 \%$ más que en el resto del país. En el caso de los trabajadores del resto de los sectores productivos de la RFN, el impacto en el salario promedio fue de $9.65 \%$.

Los resultados encontrados coinciden con los de la Comisión Nacional de Salario Mínimo (CONASAMI, 2019), la cual señala que, por la elevación de los SMF, los trabajadores en general de la RFN ganan $6.7 \%$ más que los del resto del país.

\section{Precios implícitos en el modelo insumo-producto}

Como establecimos anteriormente, existe interés en utilizar un mecanismo de trasferencia sobre los precios regionales debido a los cambios en el SMF y, con el cambio exógeno en los precios inducidos por esta propuesta de política laboral, calcular el impacto económico en variables claves para la IMMEX y la RFN.

Al respecto, la política laboral regional propone que el mecanismo de trasmisión de los efectos económicos sea por medio de la vía de cambios de precios. Es por eso que para modelar los impactos económicos, en este caso se utilizará la metodología de insumo-producto con precios implícitos con el fin de reconciliar las visiones del modelo multisectorial clásico orientadas por la oferta y por la demanda siguiendo la propuesta de Park (2006). Se entiende que el precio implícito o sombra de un bien es el precio que dicho bien alcanzaría en un mercado completamente competitivo y en ausencia de cualquier tipo de distorsión (Lee, Blakeslee y Butcher, 1977; Park, 2006; Sharify, 2013; Sharify y Sancho, 2011; Souto, 2002).

El modelo insumo-producto puede expresarse desde la perspectiva de la oferta (s) y de la demanda (d). En el primer caso, se considera la provisión de insumos primarios, es decir, el valor agregado (y sus componentes). Esta versión del modelo fue propuesta por Ghosh (1958). Y en el segundo caso, se considera 
la representación estándar del modelo insumo-producto que considera la demanda final total (y sus componentes). Esta versión del modelo corresponde a la versión original de Leontief (1953).

Aquí, el planteamiento metodológico considera que en el modelo insumo-producto por el lado de la oferta se puede representar por el conjunto de transacciones $(Z)$ como una proporción de los valores de producción $(X)$ que se obtienen en cada sector, en lo que se conoce como matriz de coeficientes técnicos y se representa con la letra A. Formalmente la matriz de Leontief queda expresada de acuerdo con:

$$
A=Z\left(\hat{X}^{s}\right)^{-1}
$$

En la expresión (1), con el acento circunflejo se representa una matriz diagonal, con la letra $s$ se invoca el enfoque de oferta y con el exponente 1 la inversa de la matriz.

Ahora, la ecuación de balance del modelo insumo-producto trata de resolver las cantidades de producción necesarias para satisfacer las necesidades de la demanda final $(Y)$ y al mismo tiempo las necesidades de insumos intermedios $(Z)$ que dependen también de los niveles de producción $(X)$, lo cual quedaría expresado de acuerdo con:

$$
X^{s^{T}}=A X^{s^{T}}+Y_{k}
$$

que tiene la solución general siguiente:

$$
X^{s^{T}}=(I-A)^{-1} Y_{k}
$$

En las expresiones (2) y (3) el supra índice T representa la transpuesta de una matriz.

El modelo de insumo-producto por el lado de la demanda puede ser representado utilizando coeficientes de distribución $\left(B=\left(\hat{X}^{d}\right)^{-1} Z\right)$ que, multiplicados por el valor de producción $(X)$ y sumados al valor agregado $(\mathrm{V})$, coinciden con los valores de producción.

$$
X^{d^{T}}=X^{d^{T}} B+u_{k}^{T} V
$$

En la expresión (4), con el supra índice T se representa la transpuesta de una matriz y, con la letra $d$, se invoca el enfoque de demanda.

La solución general del anterior modelo es la siguiente: 


$$
X^{d^{T}}=V_{l}(I-B)^{-1}
$$

En ambos casos, las soluciones generales, además de estar expresadas como en las fórmulas anteriores de acuerdo con los valores de la demanda final $(Y)$ y el valor agregado $(V)$, pueden expresarse alternativamente en términos de su incremento o de algunos de sus componentes.

En la propuesta de Park (2006) se argumenta y sustenta la reconciliación entre los modelos y la forma en que se pueden expresar los cambios en el valor agregado $(V)$ en cambios de la demanda final $(Y)$, y viceversa. Además, los cambios en el precio del valor agregado o alguno de sus componentes pueden ser expresados de acuerdo con:

$$
\Delta P=\Delta V_{l}^{p}(I-A)^{-1}
$$

mismos que deben ser ajustados por la participación del valor agregado en la producción (7) y luego ser trasformados al modelo de demanda (8), de acuerdo con:

$$
\begin{aligned}
& \Delta_{t=1} P^{Y_{k}}=\left(\hat{X}^{d}\right)^{-1} Y \Delta_{t=1} P^{d} \\
& \Delta_{t=1} \tilde{P}^{s^{T}}=\left(\hat{X}^{d}\right)^{-1} Y \Delta_{t=1} P^{d}
\end{aligned}
$$

Por último, se puede realizar un análisis de impacto económico en el valor de la producción debido a cambios en los precios exógenos que afectan la demanda final, según la siguiente ecuación:

$$
\Delta_{t=1} X^{s^{T}}=\left({ }_{t=0} X^{s^{T}}+\Delta X^{s^{T}}\right) \Delta_{t=1} \tilde{P}^{s^{T}}
$$

El cual, una vez calculado, se puede utilizar para medir los impactos económicos de la producción, del personal, del ingreso y del valor agregado, utilizando los coeficientes que relacionan esas magnitudes con la producción. El impacto del cambio de los precios exógenos a corto plazo en las anteriores variables clave a nivel de sectores de actividad será mayor cuanto mayor sean los encadenamientos hacia atrás, dados ciertos encadenamientos hacia adelante. Adicionalmente, el impacto sectorial está condicionado por el grado de competencia, las rigideces, los controles y las regulaciones.

\section{Modelo insumo-producto de la región fronteriza y del resto de México}

Para aplicar la anterior metodología, se estimó una tabla insumo-producto para la región fronteriza norte (RFN) y el resto de México (RMX) usando como base 
la tabla nacional oficial de 2013 (Instituto Nacional de Estadística y Geografía, 2014), desagregada del nivel de cuatro dígitos del Sistema de Clasificación Industrial de América del Norte (SCIAN) e integrada por 257 sectores según el SCIAN.

El procedimiento de la regionalización de la tabla nacional oficial se realizó usando un enfoque birregional. El procedimiento de regionalización se esboza de la manera siguiente. En primer lugar, la estimación de la tabla insumo-producto entre la RFN y el RMX requiere la estimación de las importaciones de la RFN desde el RMX, de tal forma que se realiza una corrección simple, por fila, de la matriz de importaciones totales de la RFN.

En segundo lugar, se efectúa un ajuste a través de un coeficiente de importaciones del RMX obtenido como el porcentaje que éstas representan sobre el total de las importaciones. La idea que reside detrás de esta corrección es asumir que la misma proporción que existe en las importaciones totales se mantiene en las intermedias. Para el caso de las exportaciones intermedias, se requiere otra serie diferente de supuestos. Además, se necesita definir un coeficiente de exportaciones intermedias de México con arreglo a los datos de comercio (disponible en el marco insumo-producto mexicano). Este coeficiente sirve para fraccionar entre intermedias y finales el total de exportaciones que la RFN efectúa para el RMX.

En tercer lugar, este vector de exportaciones intermedias se debe distribuir entre los diferentes sectores considerados. Para ello, la mejor información es aquella que aparece en la tabla doméstica mexicana, en este caso, su estructura por filas. Los dos ajustes por filas satisfacen las identidades comerciales para las importaciones y las exportaciones de la región fronteriza norte como información primaria; es decir, las exportaciones intermedias más las finales son, por definición, iguales a las totales.

Tabla 1. Matriz birregional de transacciones (billones, millardos y millones de pesos) de 2013

\begin{tabular}{|l|r|r|r|r|r|r|}
\hline & \multicolumn{2}{|c|}{ Demanda intermedia } & \multicolumn{2}{|c|}{ Demanda final } & \multicolumn{1}{c|}{$\begin{array}{c}\text { Resto del } \\
\text { mundo }\end{array}$} & $\begin{array}{c}\text { Disponibilidad } \\
\text { total }\end{array}$ \\
\hline & RFN & RMX & RFN & RMX & $\begin{array}{c}\text { Exportaciones } \\
\text { al RM }\end{array}$ & \\
\hline $\begin{array}{l}\text { Región } \\
\text { fronteriza norte }\end{array}$ & 288689 & 31337 & 505959 & 165559 & 1371913 & 2363456 \\
\hline Resto de México & 849694 & 6921965 & 793211 & 13170357 & 3543964 & 25279192 \\
\hline $\begin{array}{l}\text { Importaciones } \\
\text { del resto del } \\
\text { mundo (RM) }\end{array}$ & 51385 & 3847499 & 8430 & 1196333 & 179802 & 5283449 \\
\hline $\begin{array}{l}\text { Insumos } \\
\text { primarios }\end{array}$ & 1174149 & 14468471 & - & - & - & 15642620 \\
\hline Producción total & 2363456 & 25279192 & - & - & & 27642648 \\
\hline
\end{tabular}

Fuente: elaboración propia con base en la tabla insumo producto nacional (INEGI, 2014). 
En cuarto lugar, sólo se requieren unos ajustes a la tabla birregional relativamente simples, una vez que se tienen la tabla doméstica nacional, la tabla doméstica de la RFN y las exportaciones e importaciones intermedias que se acaban de exponer. Es importante destacar que para conseguir la consistencia global de la tabla birregional se han hecho unos ajustes finales aplicando el RAS modificado (Cárdenas, 2006). ${ }^{2}$ En la estimación del método RAS surgieron discrepancias que significaban menos de $1 \%$ del total de insumos intermedios.

En la tabla 1 se presenta la estructura fundamental y los valores del modelo birregional: RFN y resto de México (RMX). Cada elemento de la tabla está identificado por su pertenencia a una fila y a una columna de la misma, las filas indican el origen de las mercancías y las columnas el destino. Además aparecen en el mismo, el flujo de exportaciones e importaciones del resto del mundo, insumos primarios y producción total.

De la tabla 1 se desprende que las ventas y las compras sectoriales de la RFN ascienden a 288 millardos 689 millones de pesos, mientras que para el RMX suman 6 billones 921 millardos 965 millones de pesos. Las ventas de la RFN al RMX son de 31 millardos 337 millones de pesos, y las ventas del RMX a la RFN ascienden a 849 millardos 694 millones de pesos.

La demanda final autónoma agregada (consumo de los hogares, del gobierno, la inversión o las exportaciones) para la RFN suma 505 millardos 959 millones de pesos, mientras que la misma para el RMX es de 13 billones 170 millardos 357 millones de pesos debido a las importaciones de la RFN desde el RMX. Las importaciones de la RFN desde el RMX ascienden a 165 millardos 559 millones de pesos y las importaciones del RMX desde la RFN son de 793 millardos 211 millones de pesos. Las exportaciones de la RFN al resto del mundo (RM) son de 1 billón 371 millardos 913 millones de pesos y las del RMX al RM son de 3 billones 543 millardos 964 millones de pesos.

El total de los insumos primarios sectoriales de la RFN es de 1 billón 174 millardos 149 millones de pesos y para RMX es de 14 billones 468 millardos 471 millones de pesos. Las importaciones de insumos intermedios de la RFN provenientes del RM es un monto de 51 millardos 385 millones de pesos y para el caso del RMX las importaciones provenientes del RM es de 3 billones 847 millardos 499 millones de pesos.

El valor del producto producido en la RFN es de 2 billones 363 millardos 456 millones de pesos y para RMX es de 25 billones 279 millardos 192 millones de pesos.

Una vez efectuada la estimación de la tabla birregional y su correspondiente calibración de parámetros, podemos evaluar cómo el SMF genera cambios en los precios relativos sectoriales y en el nivel general de precios regionales. A su vez esto ocasiona cambios en las variables económicas clave, como la producción, el empleo de personal y el valor agregado, tanto en la IMMEX como en la RFN.

2 RAS es un método para obtener una tabla insumo-producto aproximada de los flujos comerciales del país o región (Stone, 1961). 


\section{Efectos del alza del SMF en las variables económicas clave de la IMMEX y de la RFN}

El estudio de impacto económico permitirá, a partir del uso de la tabla insumo-producto birregional, traducir el cambio de la demanda final generada por la variación de precios regionales en impactos directos e indirectos, inducidos y de propagación regional en la producción bruta, en el personal ocupado y en el valor agregado.

Más aún, los efectos económicos que se deriven del cambio exógeno de precios regionales se medirán tanto de forma sectorial (IMMEX y RFN) como de forma geográfica (RFN y RMX). Mientras que en el primer caso el modelo analiza la importancia relativa de los sectores económicos dentro de cada región; en el segundo caso, se lleva a cabo un reparto en función del peso de cada área geográfica.

En el conjunto de sectores de la tabla insumo-producto birregional desagregada a 257 sectores según el SCIAN, se decidió agregar los sectores predominantes de la IMMEX (en 4 sectores) y los sectores más representativos del resto de las actividades económicas de la RFN (en 6 sectores). Esto simplifica el análisis realizado.

Tanto la evidencia empírica del traspaso del alza de 100\% del SMF como el modelo intersectorial de precios señalan que se generará un aumento sobre la inflación general en la RFN de 2.9\%. Sin embargo, los precios sectoriales cambian también, por lo cual se midió el impacto económico en variables clave en la IMMEX y la RFN. Los resultados se muestran en la tabla 2.

Tabla 2. Impacto económico del aumento en los precios en la IMMEX y en la RFN (variación porcentual)

\begin{tabular}{|l|c|c|c|}
\hline \multicolumn{1}{|c|}{ Efecto agregado } & Producción & Empleo & Valor agregado \\
\hline \multicolumn{1}{|c|}{ Total } & 0.99 & 1.70 & 1.07 \\
\hline Actividades primarias & 0.60 & 0.95 & 0.59 \\
\hline Minería y otras secundarias & 0.82 & 1.28 & 0.58 \\
\hline Manufacturas sin predominio maquilador & 0.27 & 0.48 & 0.32 \\
\hline Maquiladoras automotrices & 0.16 & 0.22 & 0.18 \\
\hline Maquiladoras aeroespaciales & 0.04 & 0.05 & 0.04 \\
\hline Maquiladoras de dispositivos médicos & 1.12 & 1.12 & 1.12 \\
\hline Maquiladoras otras manufacturas & 0.90 & 1.18 & 1.12 \\
\hline Comercio & 0.56 & 0.56 & 0.56 \\
\hline Trasporte & 0.89 & 1.32 & 0.94 \\
\hline Servicios & 2.23 & 3.19 & 2.08 \\
\hline Maquiladora & 0.53 & 0.87 & 0.71 \\
\hline No maquiladora & 1.11 & 1.88 & 1.15 \\
\hline
\end{tabular}

Fuente: elaboración propia con datos de la matriz birregional insumo-producto: frontera norte y el resto de México. 
De la tabla se desprende que el alza en los costos laborales incrementan los costos medios y marginales de la industria regional y por lo tanto el precio de venta, lo cual provoca a su vez una disminución en el agregado de la RFN: en el nivel de la producción bruta, de aproximadamente 1\%; en la caída del personal ocupado de $1.7 \%$ y una reducción de $1.07 \%$ en el valor agregado en la RFN. Si desagregamos los resultados para la IMMEX (maquila), notamos que es menos sensible a la variación porcentual de precios con una reducción en la producción bruta de $0.53 \%$, en la caída del empleo de $0.87 \%$ y de $0.71 \%$ en el valor agregado, mientras que el resto de la RFN (no maquila) es más sensible a las variaciones de cambios de precios, con una caída en la producción bruta de $1.11 \%$, en el personal ocupado de 1.88 y en el valor agregado de $1.15 \%$.

Es importante notar que los resultados del modelo intersectorial de cambios en el valor agregado pueden estar subestimados. Es decir, existen cambios en distintos componentes del valor agregado debido a la nueva política económica federal. Por ejemplo, los impuestos en algunos sectores bajaron como resultado del cambio en la política fiscal, los sueldos de varios sectores se elevaron en respuesta a la política laboral, y los insumos y bienes importados de ciertos sectores aumentaron en términos monetarios como consecuencia de la depreciación implícita de la moneda nacional. Esto implicaría un mayor nivel de ingresos de algunos grupos sociales en la RFN.

Por otra parte, ante la existencia de rigideces en las variaciones de los costos sectoriales en la RFN, se provocará una variación negativa en la producción sectorial: $0.60 \%$ en las actividades primarias; $0.82 \%$ en la minería; $0.48 \%$ en la manufactura; $0.16 \%$ en la maquila automotriz; 0.04 en la maquila aeroespacial; $1.12 \%$ en la maquila de dispositivos médicos; $0.56 \%$ en el comercio; $0.89 \%$ en el trasporte y $2.23 \%$ en los servicios, todo debido al cambio en el SMF de algunos sectores regionales.

Por la misma razón, las variaciones en los costos de la RFN inducen una variación negativa en el personal ocupado sectorial de $0.95 \%$ en actividades primarias; $1.28 \%$ en minería; $0.27 \%$ en manufactura; $0.22 \%$ en maquila automotriz; 0.05 en maquila aeroespacial; $1.12 \%$ en maquila de dispositivos médicos; $0.56 \%$ en comercio; $1.32 \%$ en trasporte; y $3.19 \%$ en servicios. Del mismo modo, se ocasiona una variación en el valor agregado sectorial de $0.59 \%$ en actividades primarias; $0.58 \%$ en minería; $0.32 \%$ en manufactura; $0.18 \%$ en maquila automotriz; 0.04 en maquila aeroespacial; $1.12 \%$ en maquila de dispositivos médicos; $0.56 \%$ en comercio; $0.94 \%$ en trasporte; y $2.08 \%$ en servicios.

El aumento del SMF es una medida de bajos efectos inflacionarios e impactos sobre la producción, el personal ocupado y el valor agregado, tanto en la IMMEX como en la RFN.

\section{Conclusiones}

Con respecto al incremento del SMF en la RFN de 88.36 a 176.72 pesos (incremento de $100 \%$ ), se puede decir que beneficia directamente a 631 mil trabajadores asegurados que ganan menos de dos salarios mínimos diarios. De ellos, 
145 mil laboran en la IMMEX y 486 mil laboran en el resto de la economía fronteriza.

El traspaso que tiene el aumento del SMF sobre el SBC (efecto dominó) en el caso de la IMMEX es de $0.0684(6.84 \%)$ en promedio, mientras que para el resto de la economía regional es de 0.0965 (9.65\%). En otras palabras, la media del incremento del salario mínimo real de 1\% ocasiona un aumento de 0.0684 en las revisiones salariales de los trabajadores del sector formal de la economía. Luego, el aumento del SMF en 100\% ocasionará 6.84\% de elevación en las revisiones salariales de la IMMEX. De igual manera, ocasionará una ampliación de $9.65 \%$ en las revisiones salariales en la RFN.

Más aún, si se asume que el alza de $100 \%$ del SBC produce un aumento a corto plazo de $0.18 \%$ en la inflación regional 12 meses después de que dicha alza ocurra, entonces se debe pensar que habrá de originarse un alza en los precios regionales de $1.2 \%\left(0.0684^{*} 0.18\right)$ en la IMMEX y de $2.9 \%\left(0.1634^{*} 0.18\right)$ en la RFN.

El aumento del SMF es una medida de bajos efectos inflacionarios que mejora el poder adquisitivo de los que menos ganan.

Este incremento exógeno de los precios regionales se traduce a su impacto económico en producción bruta, personal ocupado y valor agregado en la IMMEX y en la RFN usando un modelo insumo-producto ampliado de precios.

El modelo insumo-producto ampliado a precios y construido para la región fronteriza norte muestra los siguientes impactos económicos. Los efectos negativos esperados para la producción bruta son de 1\%, en el personal ocupado, de $1.7 \%$, y en el valor agregado, de $1.07 \%$.

Debemos resaltar que los cálculos del modelo intersectorial de precios relativos a impacto económico en valor agregado pueden estar subestimados, debido a que existen cambios en distintos componentes de este concepto; es decir, los impuestos al valor agregado bajaron como resultado de la política fiscal decretada por el gobierno federal. Pero, contrariamente, los salarios para los trabajadores que ganaban el año anterior menos de dos salarios mínimos aumentaron por la política laboral. Consecuentemente, el efecto negativo sobre el valor agregado puede estar subestimado.

La distribución de impactos económicos en producción, personal ocupado y valor agregado a escala sectorial depende de la sensibilidad de estos cambios en los salarios. Por ejemplo, en la RFN el impacto negativo en la producción se manifiesta principalmente en las actividades primarias, que caen en $0.6 \%$, en minería $0.8 \%$, en manufactura $0.5 \%$, en comercio $-0.6 \%$, en trasporte $0.9 \%$ y en servicios $2.3 \%$. En la IMMEX el impacto recesivo se muestra en automotriz $0.04 \%$, en la aeroespacial $1 \%$ y dispositivos médicos $0.6 \%$. Luego, la IMMEX muestra menos sensibilidad al cambio en el salario mínimo.

La distribución sectorial de la variación negativa del personal ocupado para la RFN se muestra para las actividades primarias $1 \%$, minería $1.3 \% ; 0.27 \%$ en manufactura; $0.22 \%$ en maquila automotriz; 0.05 en maquila aeroespacial; $1.12 \%$ en maquila de dispositivos médicos; $0.56 \%$ en comercio; $1.32 \%$ en trasporte; y $3.19 \%$ en servicios. Así mismo ocasionan una variación en el valor agregado sectorial de $0.59 \%$ en actividades primarias; $0.58 \%$ en minería; $0.32 \%$ en manufactura; $0.18 \%$ en maquila automotriz; 0.04 en maquila aeroespacial; $1.12 \%$ 
en maquila de dispositivos médicos; $0.56 \%$ en comercio; $0.94 \%$ en trasporte; y $2.08 \%$ en servicios.

De lo anterior, podemos establecer que la política laboral regional es una medida de bajos efectos inflacionarios y de contracción, aunque ligera, en la actividad económica, pero que mejora el poder adquisitivo de los que menos ganan.

\section{Referencias}

Addison, J. T. (1999). Minimum wages and poverty. Industrial and Labor Relation Review, 52(3), 393-409.

Banco de México (Banxico). (2016). Salario mínimo e inflación. Documento preparado para la Comisión Consultiva para la Recuperación Gradual y Sostenida de los Salarios Generales y Profesionales.

Cárdenas, A. (2006). Impactos de cambios exógenos de precios de los energéticos, comparativo entre sectores productivos de Baja California; Querétaro y Nuevo León (tesis inédita de Maestría en Desarrollo Regional). El Colegio de la Frontera Norte. Tijuana, Baja California, México.

Carrillo, J., Fuentes, N. A., Brugués, A., y González-König, G. (2019). Impacto del paquete económico 2019 en la IMMEX y región fronteriza norte de México, Primer Reporte de Investigación. El Colegio de la Frontera Norte, Tijuana.

Comisión Nacional de Salario Mínimo (CONASAMI). (2019). Evaluación de impacto: efectos del aumento del salario mínimo en la Zona Libre de Frontera Norte. Gobierno de México, Dirección Técnica de CONASAMI.

Diario Oficial de la Federación. (28 de diciembre de 2018). Ley de Ingresos de la Federación para el Ejercicio Fiscal de 2019.

Ghosh, A. (1958). Input-output approach to an allocative system. Economica, $15,58-64$.

Gobierno de la Ciudad de México. (2014). Política de recuperación del salario mínimo en México y en el Distrito Federal: propuesta para un acuerdo nacional. México: Ciudad de México.

Gosling, A. (1996). Minimum wages: Possible effects on the distribution of income. Fiscal Studies, 17(4), 31-48.

Hernández, F. (2016). Comentarios: salarios mínimos e inflación (Banco de México). Centro de Investigación y Docencia Económicas (CIDE), comparecencia ante la Comisión Nacional de Salarios Mínimos.

Huesca, L., y Llamas, L. (2017). La posibilidad de mejora salarial en México: costos e impactos en la pobreza. Premio Nacional de Investigación Social y de Opinión Pública (CESOP). Cámara de Diputados, pp. 45-90.

Instituto Nacional de Estadística y Geografía (INEGI). (2014). Matriz de insumo producto. Base 2013. Sistema de Cuentas Nacionales. Recuperado de https: / /inegi.org-mx/Programas/MIP/2013

Johnson, W. R. (1983). The distributional and efficiency effects of increasing the minimum wage: a simulation. The American Economic Review, 73(1), 204- 
211.Lee, G. K., Blakeslee, L. L., y Butcher, W. R. (1977). Effects of exogenous price changes on a regional economy: an input-output analysis. International Regional Science Review, 2(1), 15-27. doi: 10.1177/016001767700200102

Lemos, S. (2004). The effect of the minimum wage on prices. Discusion Papers Series. IZA DP No. 1072.

Leontief, W. (1953). Studies in the Structure of the American Economy. Nueva York: Oxford University Press, 1953.

Lin, C., y Yun, M.-S. (2016). The effects of the minimum wage on earnings inequality: Evidence from China. IZA Discussion Papers, No. 9715.

MaCurdy, T., y O'Brien-Strain, M. (2000). Increasing the Minimum Wage: California's Winners and Losers. San Francisco: Public Policy Institute of California.

Martínez, G. (14 de febrero de 2019). Revisan que la industria cumpla con el alza salarial. El Economista.

Moreno-Brid, J. C.; Garry, S. y Monroy-Gómez Franco, L. A. (2014). El salario mínimo en México. EconomíaUNAM, 11(33), 78-93.

Neumark, D. (2002). Do minimum wages fight poverty? Economic Inquiry, 40(3), 315-333.

OIT-Grupo Salario (mayo 24 de 2016). El efecto del salario mínimo (efecto dominó/efecto faro) en la distribución salarial de la economía mexicana. Documento final.

Park, J. (2006). The Supply-Driven Input-Output Model: A Reinterpretation and Extension. Los Ángeles, California: University of Southern California, School of Policy, Planning and Development.

Salazar, S., Amador, J. y Serrano, C. (2019). El aumento del salario mínimo: una medida sin efectos inflacionarios que mejora el poder adquisitivo de los que menos ganan. Análisis Económico BBVA Research.

Secretaría de Economía. (2011). Decreto para el fomento de la industria manufacturera, maquiladora y servicios de exportación (IMMEX). Diario Oficial de la Federación, 24 de diciembre de 2010.

Secretaría de Hacienda y Crédito Público. (2018). Paquete económico para el ejercicio fiscal 2019. Gobierno Federal. Disponible en https://www.ppef. hacienda.gob.mx/es/PPEF2019

Sharify, N. (2013). Input-output modelling of the effect of implicit subsidies on general prices. Economic Modeling, 33, 913-917. doi:10.1016/j. econmod.2013.06.011

Sharify, N., y Sancho, F. (2011). A new approach for the input-output price model. Economic Modelling, 28(1-2), 188-195.

Souto, G. (2002). Los precios sombra en España a partir del análisis input output. Barcelona: Universidad Autónoma de Barcelona, Departamento de Economía Aplicada. 